YITP-04-50

DESY-04-179

\title{
A study of late decaying charged particles at future colliders
}

\author{
Koichi Hamaguchi, ${ }^{1}$ Yoshitaka Kuno, ${ }^{2}$ Tsuyoshi Nakaya, ${ }^{3}$ and Mihoko M. Nojiri ${ }^{4}$ \\ ${ }^{1}$ Deutsches Elektronen-Synchrotron DESY, 22603 Hamburg, Germany \\ ${ }^{2}$ Department of Physics, Graduate School of Science, \\ Osaka University, Toyonaka, Osaka 560-0043, Japan \\ ${ }^{3}$ Department of Physics, Kyoto University, Kyoto , Japan \\ ${ }^{4}$ YITP, Kyoto University, Kyoto 606-8502, Japan
}

(Dated: February 2, 2008)

\begin{abstract}
In models where the gravitino is the lightest supersymmetric particle (LSP), the next-to-lightest supersymmetric particle (NLSP) is long-lived. We consider an important charged NLSP candidate, the scalar tau $\tilde{\tau}$. Slow charged NLSPs may be produced at future colliders and they may be stopped in a massive stopper which simultaneously serves as a detector for the NLSP and its decay products. We found the number of events at a 1 kton to $\mathrm{O}(10)$ kton detector could be significant enough to study the NLSP decays with lifetime shorter than $10^{10} \mathrm{sec}$ at the LHC. The performance of existing 1 kton detectors may be good enough to do such studies at the LHC, if they can be placed close to the ATLAS/CMS detectors. At a future $e^{-} e^{-}$collider, scalar electrons $\tilde{e}^{-} \mathrm{s}$ are copiously produced. Slow NLSPs may be produced from the $\tilde{e}^{-}$decay. The number of stopped NLSPs at a future linear collider could be large enough to study rare decay modes of the NLSP.
\end{abstract}

PACS numbers: 


\section{INTRODUCTION}

Supersymmetry (SUSY) is one of the key features of theories of quantum gravity, such as superstring. Supersymmetric extension of gravity (supergravity), or local SUSY, predicts a spin-3/2 particle, the gravitino. When supersymmetry is spontaneously broken, the gravitino acquires a mass by absorbing a massless goldstino. In most of the supersymmetric models, SUSY is broken in a hidden sector. While the masses of superpartners of the standard model (SM) particles may depend on the mechanism to mediate SUSY breaking in the hidden sector, the gravitino mass is only described by the SUSY breaking scale, $F$, and the Planck scale, $M_{\mathrm{P}}$, as $m_{3 / 2}=F /\left(\sqrt{3} M_{\mathrm{P}}\right)$. Discovery of the gravitino would be therefore one of the most direct ways to prove the nature of the hidden sector of the supersymmetric models.

The rate of the direct production of a gravitino from particle collisions is small. If the gravitino is the lightest supersymmetric particle (LSP), it is produced from cascade decays of heavier sparticles, and its nature can be thus studied. The LSP gravitino is also cosmologically important. The reheating temperature after inflation is severely constrained by the 'overclosure' problem [1]. There have been, however, proposed several mechanisms which avoid this constraint and which, in addition, explain the cold dark matter density

in terms of thermally produced LSP gravitinos [2]. Late time decays of next-to-lightest supersymmetric particles (NLSPs) might change the predictions of big bang nucleosynthesis $(\mathrm{BBN})$ if the lifetime is longer than $\mathrm{O}(1)$ sec [3], which leads to constraints on the masses of NLSP and gravitino 4], and parameters of supersymmetric models [5]. Gravitino dark matter scenario may also be realized non-thermally, e.g. via NLSP decays [6] or right-handed sneutrino decay [7].

In the LSP gravitino scenario, the NLSP is metastable, and they escape detector before decaying into the gravitino. The NLSP is likely the scalar tau lepton $\tilde{\tau}$ (stau), because the slepton masses receive smaller radiative corrections from gaugino masses than squarks, and the stau mass receives a larger Yukawa correction than the other sleptons. The lighter mass eigenstate $\tilde{\tau}$, which is a linear combination of $\tilde{\tau}_{L}$ and $\tilde{\tau}_{R}$, becomes even lighter due to the left-right mixing. The collider phenomenology has been studied in Refs. [8, 9, 10, 4] .

The main decay mode of the NLSP stau is $\tilde{\tau} \rightarrow \tau \psi_{3 / 2}$, where $\psi_{3 / 2}$ is the gravitino. The decay width depends only on the stau mass $m_{\tilde{\tau}}$, the gravitino mass $m_{3 / 2}$, and the Planck 
scale $M_{\mathrm{P}}$. It is given by the following formula

$$
\Gamma_{\tilde{\tau}}\left(\tilde{\tau} \rightarrow \psi_{3 / 2} \tau\right)=\frac{m_{\tilde{\tau}}^{5}}{48 \pi m_{3 / 2}^{2} M_{\mathrm{P}}^{2}}\left(1-\frac{m_{3 / 2}^{2}}{m_{\tilde{\tau}}^{2}}\right)^{4},
$$

where the tau mass is omitted.

The lifetime of the $\operatorname{NLSP}\left(\tau_{\mathrm{NLSP}}=1 / \Gamma_{\tilde{\tau}}\right)$ could be long because the decay width is suppressed by $M_{\mathrm{P}}^{-2}$. The lifetime is sensitive to the yet unknown gravitino mass. For $\tilde{\tau}$ mass of $100 \mathrm{GeV}$, the lifetime of NLSP stau would be 60 nsec for a gravitino of $m_{3 / 2}=1$ $\operatorname{keV}\left(F=\left(2 \times 10^{6} \mathrm{GeV}\right)^{2}\right)$, and $6 \times 10^{6} \sec$ for $m_{3 / 2}=10 \mathrm{GeV}\left(F=\left(6 \times 10^{9} \mathrm{GeV}\right)^{2}\right)$. If we can determine the gravitino mass by measuring the energy of the emitted tau lepton, the measurement of the stau lifetime $\left(\Gamma_{\tilde{\tau}}\right)^{-1}$ leads to a determination of a "supergravity Planck scale", $M_{\mathrm{P}}[$ supergravity $]=M_{\mathrm{P}}\left(m_{\tilde{\tau}}, m_{3 / 2}, \Gamma_{\tilde{\tau}}\right)$ [11]. It would be a crucial test of supergravity by comparing it with the macroscopically determined Planck scale of Einstein gravity, $M_{\mathrm{P}}$ [gravity] $=(8 \pi G)^{-1 / 2}=2.43534(18) \times 10^{18} \mathrm{GeV}$ [12]. On the other hand, in the case where the gravitino mass can not be directly determined due to limited experimental resolutions, its mass, or SUSY breaking scale, can be estimated from the lifetime in the assumption that Eq. (11) is correct. Furthermore, a study of a rare 3-body decay, $\tilde{\tau} \rightarrow$ $\tau \gamma \psi_{3 / 2}$, can reveal the peculiar form of the gravitino couplings, and may even determine the gravitino spin of $3 / 2$ [11].

Previously, the determination of the charged NLSP (CNLSP) lifetime has been studied by many authors and it was found that the CNLSP decay is detectable for $0.5 \mathrm{~m}<c \tau_{\mathrm{CNLSP}}<$ $1 \mathrm{~km}$ [10]. In this paper, we study the possibility to measure the CNLSP lifetime and decay patterns for the case when it is long-lived so that the prompt decay inside the detector can be neglected. For the CNLSP with sufficiently slow velocity, it can be stopped in the stopper material placed outside the main detector after losing its energy from ionization in the main detector materials. ${ }^{1}$ Then the lifetime of CNLSP thus stopped would be measured by detecting subsequent decay of the CNLSP in the stopper. The stopper needs to be a tracking detector to measure the stopping position and timing of the parent CNLSP and also the momenta, positions and timings of the charged particles and photons arising from the CNLSP decay. The requirements of the stopper detector are also discussed. Such study,

\footnotetext{
${ }^{1}$ For an earlier proposal to collect long-lived charged heavy fermions, see Ref. [13. Although details was not given, an idea of placing a stopping material outside the main detector to collect the CNLSPs has been pointed out in Ref. 14].
} 
if possible at future colliders such as the LHC, will also reveal the important interactions that may affect the big bang nucleosynthesis in the early universe.

This paper is organized as follows. In section 2, we discuss the requirements to study the CNLSP decay. In section 3, we discuss a possible study of the CNLSP at the LHC (ATLAS/CMS) 15, 16, 17]. The velocity distribution of CNLSP $\left(\beta_{\tilde{\tau}}\right)$ at the LHC is shown

at a sample point of the gauge mediation model [18], which is studied in the ATLAS technical design report. The number of CNLSPs stopped in a $\mathrm{O}(1)$ kton stopper is estimated, and it is found to be as large as $\mathrm{O}(5000)$ events for an integrated luminosity of $300 \mathrm{fb}^{-1}$ and squark masses of $m_{\tilde{q}} \sim 700 \mathrm{GeV}$. So that, one can determine a lifetime of the CNLSP of $\tau_{\text {CNLSP }}<1000$ years or so, which corresponds to the SUSY breaking scale as large as $\mathrm{O}\left(10^{10}\right) \mathrm{GeV}$, or gravitino mass of $\mathrm{O}(100) \mathrm{GeV}$. Here, we also discuss a possibility to make use of existing detector, taking Soudan 2 as an example. In section 4, we will study the case at proposed future linear colliders [19]. The direct production of the CNLSP $\tilde{\tau}$ at a $e^{+} e^{-}$collider may not be efficient because the cross section is suppressed by $\left(\beta_{\tilde{\tau}}\right)^{3}$, while one wants to keep $\beta_{\tilde{\tau}}$ relatively small so that CNLSP can be easily stopped. When the mass difference between $\tilde{e}$ and $\tilde{\tau}$ is small, an alternative option of $e^{-} e^{-}$collision at the $\tilde{e}^{-} \tilde{e}^{-}$ threshold energy is more attractive, since the production cross section is only suppressed by $\beta_{\tilde{e}}$. It is found that the number of the CNLSPs stopped in the stopper could be as large as $10^{5}$. If this is the case, one can study not only the lifetime of the CNLSP, but also the branching ratios and decay distributions of various CNLSP decay modes, including the rare decay modes.

\section{PRINCIPLE OF LIFETIME MEASUREMENTS OF STOPPED CNLSPS}

We first discuss how we could measure the lifetime of the CNLSP, which can be produced at collider experiments. The CNLSP would be metastable and long-lived. Its lifetime $\tau_{\mathrm{CNLSP}}$ might range from $\mathrm{O}(100)$ nsec to $\mathrm{O}(1000)$ years, depending on the CNLSP and gravitino masses. When $c \tau_{\mathrm{CNLSP}}$ is too long to decay frequently in the detector ( $\left.\gtrsim 1000 \mathrm{~ns}\right)$, its lifetime cannot be measured directly at the main detector of collider experiments. However the CNLSPs with low velocity lose their kinetic energy by ionization loss, since they are charged, and could be stopped at a massive stopper material, which can possibly be placed outside the main detector. A large number of the CNLSPs thus stopped could be stored and 
accumulated in the stopper for a significantly long time period. The CNLSPs then decay after $t \sim \tau_{\mathrm{CNLSP}}$.

The stopper should be an active detector with good tracking capability to measure the lifetime of the CNLSP. First of all, it should measure the ionization per unit length $-d E / d x$ to reject a $\mu$ track from that of signal CNLSP [8]. The stopping timings $t_{\text {stop }}$ and positions of all the CNLSP candidates are measured and recorded one by one, as well as the CNLSP decay time $t_{\text {decay }}$ and its decay products. The lifetime of CNLSP is obtained from the distribution of a timing difference $t_{\text {decay }}-t_{\text {stop }}$. In particular, to correspond the parent CNLSP and daughter decay products, the requirement that the decay products originate from the stopping position of the parent CNLSP is of crucial importance. Therefore, the stopper has to have a sufficiently high position resolution, as well as a fine detector segmentation.

The stopper should be massive so that as large number of CNLSPs as possible can be stopped, of the order of $\mathrm{O}(1) \sim \mathrm{O}(10)$ kton. Typically, we need to have, at least, $\mathrm{O}(10)$ CNLSPs stop and decay/year inside the stopper detector to estimate the lifetime in accuracy of a few $10 \%$. When a huge number of CNLSPs can be accumulated, the distributions of the decay products can be studied. The CNLSP is likely to be a $\tilde{\tau}$ and it decays into a $\tau$ lepton. The $\tau$ further decays into $e^{-}, \mu^{-}, \pi^{-}, \rho^{-}, a_{1}$ and so on. $\rho^{-}$and $a_{1}^{-}$further decay into $\pi^{-}$and $\pi^{0}$ ('s), and momenta of the decay products are parallel. The decay distributions contain physics information. The stopper detector might have good calorimetric performance for them, then the gravitino mass can be calculated from the CNLSP mass and the tau energy, which is estimated from the end point of the tau jet energy distribution. The composition of the $\tau$ jet is also important. The tau lepton is generally polarized depending on the left-right mixing of $\tilde{\tau}$, and the polarization of $\tau$ can be studied by measuring the $E_{\gamma} / E_{\text {jet }}$ ratio [20]. In general, the $\pi^{ \pm}$is detected as an ionizing track starting from the decay point and it ends by interacting hadronically after penetrating $\sim 80 \mathrm{~g} / \mathrm{cm}^{2}$. On the other hand, $\gamma$ 's are converted into EM showers after one radiation length $\left(\mathrm{O}(10) \mathrm{g} / \mathrm{cm}^{2}\right)$. Ideally, the detector needs to be segmented into cells with a thickness less than $\mathrm{O}(10) \mathrm{g} / \mathrm{cm}^{2}$ to any direction, so that $E_{\gamma} / E_{\text {jet }}$ can be measured.

To estimate a suitable velocity $(\beta)$ of the CNLSP to stop, a range $R$, which is a distance for a charged particle to penetrate before stopping, is evaluated based on the Bethe-Bloch equation. For the value of the $\beta$ of interest, the range $R$ is described by a function of $\beta \times \gamma\left(\gamma=1 / \sqrt{1-\beta^{2}}\right)$ with a mild dependence on types of the stopper material. For 
iron, $R / M=10 \mathrm{~g} /\left(\mathrm{cm}^{2} \cdot \mathrm{GeV}\right)$ is necessary for a particle with $\beta \gamma=0.43, R / M=30$ $\mathrm{g} /\left(\mathrm{cm}^{2} \cdot \mathrm{GeV}\right)$ for $\beta \gamma=0.62$, and $R / M=50 \mathrm{~g} /\left(\mathrm{cm}^{2} \cdot \mathrm{GeV}\right)$ for $\beta \gamma=0.75$ [12], where $M$ is the mass of a charged particle. The CNLSP with $\beta \gamma>1$ needs to go through $>10000$ $\mathrm{g} / \mathrm{cm}^{2}$ when $m_{\mathrm{CNLSP}}=100 \mathrm{GeV}$. It is difficult to stop such relativistic particles, therefore we will concentrate on the CNLSPs with slow velocity.

It is noted that most of the main detectors for collider experiments have significant thickness. Typically thickness of the detector is around 10 absorption length $\sim 1000 \mathrm{~g} / \mathrm{cm}^{2}$ for pseudo-rapidity ${ }^{2}|\eta|<1$ (see [15]). They serve as a degrader to reduce kinetic energy of the CNLSPs before reaching the stopper. Some of the CNLSPs also stopped inside the detector, however, those stopped inside the detectors cannot be used to study their decay unless the detectors are significantly modified. While most of those CNLSPs will stop in the calorimeter, the granularity of the hadronic calorimeters might not be fine and positions where they stopped are not precisely determined. ${ }^{3}$ It is then not obvious to reject an activity in the calorimeter from the cosmic ray events. Furthermore, those detectors take data only at the time of collisions. The decay of the particles with a lifetime longer than the bunch spacing will not be tagged.

In the next subsequent sections, the velocity distribution of the CNLSPs and the number of the CNLSPs stopped in the stopper are estimated, together with accuracy of lifetime measurement, for the LHC and future linear colliders separately.

\section{THE CNLSP STUDIES AT HIGH LUMINOSITY OPTION OF THE LHC.}

At the LHC, the CNLSP can be produced from cascade decays of squarks $\tilde{q}$ and gluinos $\tilde{g}$. The production cross section is sensitive to the squark and gluino masses. If $m_{\tilde{q}} \sim$ $m_{\tilde{g}} \sim 700 \mathrm{GeV}(1 \mathrm{TeV}), \sigma_{\mathrm{SUSY}}=20(3) \mathrm{pb}$ and $\sim 10^{7}\left(10^{6}\right)$ CNLSP will be produced for $\int d t \mathcal{L}=300 \mathrm{fb}^{-1}$. For the case where $\tilde{q}$ and $\tilde{g}$ are as heavy as $\sim 2 \sim 3 \mathrm{TeV}$, one may still search for the CNLSPs model independently from their direct productions which is discussed

in [4], however, the study of the CNLSP decays will be limited statistically. Therefore we concentrate on the case where CNLSPs are produced dominantly from $\tilde{q}$ and $\tilde{g}$ decays.

\footnotetext{
${ }^{2} \cos \theta=\tanh \eta$, where $\theta$ is the angle from the beam direction.

${ }^{3}$ For example, ATLAS hadronic calorimeter, whose internal radius is $2.28 \mathrm{~m}$, has position resolution $\Delta \eta \times$ $\Delta \phi=0.1 \times 0.1$. In the radial direction, it is segmented into (only) three layers.
} 
A case where the NLSP is a charged slepton is studied in paper [22] at a gauge mediation model point $\mathrm{G}^{2} \mathrm{~b}^{4}$ by using ISAJET [21]. At this point, gluino decays dominantly into a squark and jet. A squark decays dominantly into gaugino like neutralinos $\tilde{\chi}_{1(2)}^{0}$ or chargino $\tilde{\chi}_{1}^{ \pm}$. The neutralinos and chargino further decay into lighter sleptons $\tilde{l}_{R}$;

$$
(\tilde{g}) \rightarrow \tilde{q} \rightarrow \tilde{\chi}_{1(2)}^{0}, \tilde{\chi}_{1}^{+} \rightarrow \tilde{l}
$$

The relevant masses of the SUSY particles at G2b are as follows; $m_{\tilde{\chi}_{1}^{0}}=112 \mathrm{GeV}, m_{\tilde{\chi}_{2}^{0}}=$ $203 \mathrm{GeV}, m_{\tilde{g}}=709 \mathrm{GeV}, m_{\tilde{u}_{L}}=685 \mathrm{GeV}, m_{\tilde{u}_{R}}=664 \mathrm{GeV}, m_{\tilde{e}_{R}}=102 \mathrm{GeV}, m_{\tilde{\tau}}=101 \mathrm{GeV}$. More details will be found in [22] and [16].

The measurement of the slepton mass is performed by using momentum measurement in inner tracker and time information at muon system. The mass error of the slepton would be around $0.1 \mathrm{GeV}$ at this point 22 ]. This means that the gravitino mass $m_{3 / 2}$ is determined with the additional $\tau_{\mathrm{CNLSP}}$ measurement by using Eq. (11).

The typical transverse momentum of the CNLSP at the LHC are determined by the mass difference between $\tilde{q}$ and $\tilde{\chi}_{i}^{0}$ or $\tilde{\chi}_{i}^{ \pm}$. The momentum of a $\tilde{\chi}$ from a stopped squark is expressed as

$$
\left|\overrightarrow{\mathrm{p}}_{\tilde{\chi}}^{\mathrm{rest}}\right|=\frac{m_{\tilde{q}}^{2}-m_{\tilde{\chi}}^{2}}{2 m_{\tilde{q}}} .
$$

The $\tilde{q}$ production is dominated by the threshold production because the parton distribution is soft. Therefore the typical absolute momentum of $\tilde{\chi}$ peaks at $\left|p^{\text {rest }}\right|$, but it is boosted by parent $\tilde{q}$ velocity. The distribution therefore depends on the decay patterns and mass spectrum. The momentum of a $\tilde{l}$ from a $\tilde{\chi}$ decay is similar to the $\tilde{\chi}$ momentum when the mass differences between $\tilde{\chi}$ and $\tilde{l}$ are small. To be quantitative, we generated $10^{5}$ SUSY events at the G2b point by using HERWIG [23], which corresponds to $\int d t \mathcal{L}=5 \mathrm{fb}^{-1}$ at the LHC. In Fig. 1, we show the $\beta \gamma$ distribution of $\tilde{l}$ at the G2b point. The dotted line shows the distribution of all events, while the solid line shows the event distribution with $\eta<1$. The typical momentum is $\beta \gamma=|\mathrm{p}| / m \sim 2$, which is consistent with the estimate based on Eq. (3). From Fig. 11, we can see the events are likely to be forward when $\beta \gamma$ is large, while

\footnotetext{
${ }^{4}$ The model point is outdated because the higgs mass is lighter than LEP II constraint. Taking $\tan \beta=20$ keeping other gauge mediation parameter, the higgs mass can be raised above the limit. We study the G2b point, because mass resolutions have been investigated in detail at this point. The mass of sleptons are very degenerated for $\tan \beta=5$ so that $\tilde{e} \rightarrow \tilde{\tau} \tau e$ is closed and $\tilde{e}_{R}$ and $\tilde{\mu}_{R}$ are long-lived. Therefore we show distribution for all sleptons in Fig. 1.
} 


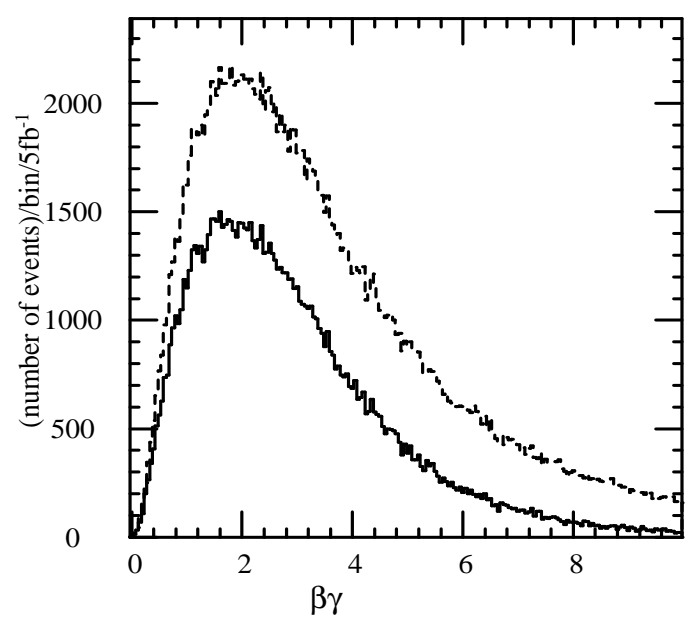

FIG. 1: The $\beta \gamma$ distribution of $\tilde{l}$ for $10^{5}$ SUSY events at the point G2b. The solid histogram shows the distribution with $\left|\eta_{\tilde{l}}\right|<1$ and the dashed histogram shows all $\tilde{l}$ distributions.

\begin{tabular}{|c|c|c|}
\hline CNLSP velocity & number of events & $|\eta|<1$ \\
\hline$\beta \gamma<0.43$ & $1.68 \times 10^{3}$ & $1.28 \times 10^{3}$ \\
$0.43 \leq \beta \gamma<0.62$ & $2.85 \times 10^{3}$ & $2.14 \times 10^{3}$ \\
$0.62 \leq \beta \gamma<0.75$ & $2.85 \times 10^{3}$ & $2.09 \times 10^{3}$ \\
\hline
\end{tabular}

TABLE I: Numbers of the CNLSP for different velocity regions at the point G2b for $10^{5}$ SUSY events $\left(5 \mathrm{fb}^{-1}\right)$ at the LHC.

the event distribution is more spherical when $\beta \gamma \sim 0$.

In Table I the number of events for a few representative $\beta \gamma$ intervals for $10^{5}$ SUSY events are shown. We found that the $0.8 \%$ of the total CNLSP (twice of the produced SUSY events) has $\beta \gamma<0.43$. Because the mass of CNLSP is about $100 \mathrm{GeV}$ for the G2b point, $R \sim 1000 \mathrm{~g} / \mathrm{cm}^{2}$ for $\beta \gamma<0.43$ and they will be stopped in the LHC detectors. We also found that $1.4 \%$ of the total CNLSPs have $0.43 \lesssim \beta \gamma<0.62$ or $0.62 \lesssim \beta \gamma<0.75$.

The expected number of the stopped CNLSP for a fixed stopper weight is inversely proportional to the square of distance of the stopper from the interaction point $R_{\mathrm{IP}}$. Note that the ATLAS (CMS) detector has a cylindrical shape whose diameter is 20m (15m) respectively. To cover the ATLAS or CMS detector with $2000 \mathrm{~g} / \mathrm{cm}^{2}$ stopper over the length of $2 R_{\mathrm{IP}}$ (corresponding to $|\eta| \lesssim 1$ ), we need more than $25 \times\left(R_{\mathrm{IP}} / 10 \mathrm{~m}\right)^{2}$ kton stoppers. 
Apparently $R_{\mathrm{IP}}$ should be larger than the half of the diameter at least.

The expected number of the trapped CNLSP at the G2b point for integrated luminosity of $300 \mathrm{fb}^{-1}$ (3 year in high luminosity) is given as a function of the stopper weight $M_{T}$ and $R_{\mathrm{IP}}$ as

$$
N(\mathrm{G} 2 \mathrm{~b})=0.51 \times 10^{4}\left(\frac{M_{T} / 1 \mathrm{kton}}{\left(R_{\mathrm{IP}} / 10 \mathrm{~m}\right)^{2}}\right) .
$$

Here we have assumed that, if $M_{T}<25\left(R_{\mathrm{IP}} / 10 \mathrm{~m}\right)^{2} \mathrm{kton}$, the fraction of the solid angle of the stopper from the IP would be reduced while keeping the thickness $2000 \mathrm{~g} / \mathrm{cm}^{2}$.

We now estimate the level of the background expected in $\mathrm{O}(1 \mathrm{kton})$ stopper at the LHC. A possible source of the background is events from the charged current interaction of the atmospheric neutrinos in the stopper. They are observed as charged particle emissions contained in the stopper, just like the stopped CNLSP decays.

The background rate can be estimated by the event rate in Super Kamiokande. In [24], the number of multi $\mathrm{GeV}$ and partially contained events (with visible energy $E_{\mathrm{vis}}>1.33 \mathrm{GeV}$ ) in Super Kamiokande is reported as 2256 events for 76 kton year. On the other hand, the energy of leptons and jets are typically of the other of $10 \mathrm{GeV}$ for the decaying CNLSP, and hence a significant part of the atmospheric neutrino background will be removed by requiring $E_{\mathrm{vis}}>10 \mathrm{GeV}$ without losing too many signals. ${ }^{5}$

The number of background events may be calculated from the neutrino energy distribution calculated in 25]. The flux is roughly proportional to $1 / E_{\nu}^{3}$ or less for $1 \mathrm{GeV}<E_{\nu}<$ $1000 \mathrm{GeV}$, while the neutrino-interaction cross section increases proportional to $E_{\nu}$. The rate of the charged current atmospheric neutrino events therefore scales as $1 / E_{\nu}^{2}$. When $E_{\mathrm{vis}}>10 \mathrm{GeV}$ is required, the rate would be reduced by a factor of $\sim 1 / 100$ compared to that for $E_{\mathrm{vis}}>1.33 \mathrm{GeV}$, so that the number of atmospheric neutrino events would be less than $1 /$ kton/year. Furthermore, if we record the positions where CNLSPs stopped, the number of background would be reduced down to a negligible level.

Another source of background is the high energy neutrinos from $p p$ collisions. The heavy quarks $b$ and $c$ are produced with very high rates, and their semi-leptonic decays produce high energy neutrinos. There are productions of $W$ bosons as well, and their decays produce neutrinos with $p_{T} \sim 45 \mathrm{GeV}$. The $\nu_{\mu}$ flux must be comparable to the muon flux at the LHC,

\footnotetext{
${ }^{5}$ High energy $\mu$ s penetrate stoppers, and one needs magnet to measure the energies. One might have to veto $\mu$ like events to reduce the atmospheric backgrounds.
} 
as a high energy $\nu_{\mu}$ is always produced with a $\mu$ production.

The production cross section of $\mu$ from various sources are plotted as the function of $p_{T}$ in Fig 2.1 in [26]. The dominant source of $\mu$ production is $b \rightarrow \mu$ for $p_{T}<35 \mathrm{GeV}$ and $d \sigma / d p_{T}(b \rightarrow \mu)=10^{-1} \mu \mathrm{barn} / \mathrm{GeV}$ for $p_{T}=10 \mathrm{GeV}, 10^{-2} \mu$ barn $/ \mathrm{GeV}$ for $p_{T}=20 \mathrm{GeV}$, and $10^{-3} \mu \mathrm{barn} / \mathrm{GeV}$ for $p_{T}=30 \mathrm{GeV}$. For $p_{T} \sim 40 \mathrm{GeV}$ the dominant $\mu$ production comes from $W \rightarrow \mu$ and $d \sigma / d p_{T}(W \rightarrow \mu) \sim 10^{-3} \mu \mathrm{barn} / \mathrm{GeV}$ at the peak. We assume the total neutrino flux is $10^{-1} \mu$ barn for $p_{T}>20 \mathrm{GeV}$ because we need to take into account $\nu_{e}$ and $\nu_{\tau}$ as well. Roughly $3 \times 10^{10}$ hard neutrinos are produced for $300 \mathrm{fb}^{-1}$. The charged current cross section of nucleon and neutrino is given in [12] as $\sigma / E_{\nu} \sim 0.677 \times 10^{-38} \mathrm{~cm}^{-2}$. The number of neutrinos which interact with 1 kton stopper at $10 \mathrm{~m}$ from the IP is less than 0.1 and can be neglected compared with atmospheric events.

There might be other source of backgrounds such as neutrons from the surrounding environment and we need detailed studies to estimate the total background rate. The position measurements of the CNLSP will provide the good signal and background discriminations.

The number of CNLSPs decaying in the detector per year depends on the lifetime of the CNLSP. When $\tau_{\text {CNLSP }}=100$ and 1000 years, the number of the events decaying in the detector/year are $1 \%$ and $0.1 \%$ of the total number of events stopped, respectively. To have 10 CNLSP decays in the 1 kton detector, the lifetime must be shorter than 1000 years for the G2b point when 1kton detector can be placed at $R_{\mathrm{IP}}=10 \mathrm{~m}$.

When sparticle masses are higher than those of the G2b point, the number of stopped CNLSPs would be significantly smaller. First of all, the squark and gluino production cross sections decrease very quickly with increasing squark mass. At the G2b point where $m_{\tilde{g}}$ and $m_{\tilde{q}} \sim 700 \mathrm{GeV}$, the total SUSY production cross section is calculated by HERWIG as 19.3 pb. On the other hand, at a model point SPS7 considered in Snowmass study [27], $m_{\tilde{g}} \sim 926 \mathrm{GeV}$ and the SUSY production cross section is $5.2 \mathrm{pb}$. The CNLSP is $\tilde{\tau}$ with $m_{\tilde{\tau}}=120 \mathrm{GeV}$ for SPS7. The number of the CNLSPs with $0.43 \leq \beta \gamma<0.62$ and $|\eta|<1$ is 2706 for $10^{5}$ produced SUSY particles. The stopper to stop all the slepton with $|\eta|<1$ and this $\beta \gamma$ range must have a weight $\sim 30 \times\left(R_{\mathrm{IP}} / 10 \mathrm{~m}\right)^{2}$ kton, because $m_{\tilde{\tau}}$ is larger. This means

$$
N(\mathrm{SPS} 7)=0.14 \times 10^{4}\left(\frac{M_{T} / 1 \mathrm{kton}}{\left(R_{\mathrm{IP}} / 10 \mathrm{~m}\right)^{2}}\right) .
$$

The number of stopped events is still large enough to measure $\tilde{\tau}<100$ years, corresponding to $m_{3 / 2} \lesssim 80 \mathrm{GeV}$. 
So far we have discussed the conditions to study CNLSP decay at the LHC. We now turn to the question if it is possible to construct a detector which satisfies these conditions. Here we take Soudan 2 detector as an example detector and consider if it can be operated close to the LHC detectors. The Soudan 2 is a fine-grained tracking calorimeter detector for proton decay searches 28]. The detector consists of 224 modules with each size $2.7 \mathrm{~m} \times 1 \mathrm{~m} \times 1 \mathrm{~m}$. A module then consists of drift tubes separated by $14.7 \mathrm{~mm}$ between steel sheets. The steel sheets are source of protons for the experiment, but they could also work as the stoppers to stop the CNLSPs. The total weight of the detector modules is $0.96 \mathrm{kton}$. The track resolution is $0.18 \mathrm{~cm} \times 0.18 \mathrm{~cm} \times 1 \mathrm{~cm}$, which is small enough to isolate each stopped CNLSP.

There will be a few $\mathrm{Hz} / \mathrm{cm}^{2}$ of charged particles at the muon system of the ATLAS detector at $\eta \sim 0$ [26]. The dead time of the drift tube of Sudan 2 is $100 \mu$ sec. The number of muons which goes through a drift tube per the drift time (dead time) must be much less than one, so that the CNLSP tracks can be reconstructed. Each tube covers $147 \mathrm{~cm}^{2}$. The expected number of charged particles/sec/tube is therefore much less than $0.1 /(100 \mu \mathrm{sec})$, even at the surface of the LHC detectors.

The energy threshold of Soudan 2 detector is $\mathrm{O}(100) \mathrm{MeV}$ for both muons and electrons. The threshold is set for proton decay study. If new detectors can be built for the CNLSP study, the ratio (stopper mass)/(number of drift tubes) may be increased while keeping most of the important aspects as the detector for CNLSP decay products.

A more realistic study on the condition in the ATLAS/CMS cabin is needed to judge if it is a realistic option to study the CNLSP decays. We stress that it is worth to investigate the possibility to start an experiment to stop and collect CNLSPs as early as possible, perhaps with the high luminosity operation of the LHC, if the CNLSPs are accessible at the low luminosity run of the LHC and the CNLSP is metastable.

\section{THE CNLSP STUDY AT $e^{-} e^{-}$LINEAR COLLIDER}

As we have discussed in the previous sections, the velocity of the stau, $\beta_{\tilde{\tau}}$, should be small enough to be stopped in the stopper. A crucial advantage for a linear collider (LC) is that one can restrict the velocity of produced particles by adjusting the beam energy.

At a $e^{+} e^{-}$collider, the direct production of a $\tilde{\tau}^{+} \tilde{\tau}^{-}$pair is suppressed near the threshold, since the cross section decreases as $\sim \beta_{\tilde{\tau}}^{3}$. On the other hand, at a $e^{-} e^{-}$collider, a slepton 


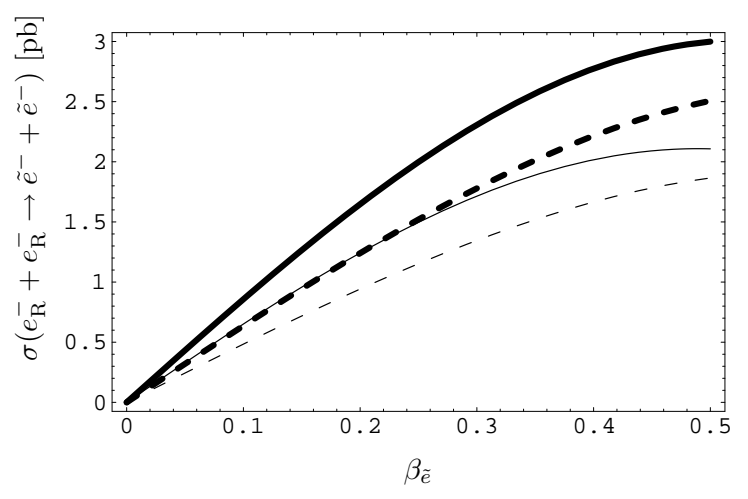

FIG. 2: Cross section $\sigma\left(e_{R}^{-}+e_{R}^{-} \rightarrow \tilde{e}^{-}+\tilde{e}^{-}\right)$for $m_{\tilde{e}}=170 \mathrm{GeV}$. We assume $100 \%$ polarization for the $e^{-}$beam. $M_{1}=180 \mathrm{GeV}$ (solid lines) and $M_{1}=300 \mathrm{GeV}$ (dashed lines). The thick lines are the total cross section and the thin lines are for selectrons with $|\eta|<1$.

pair of $\tilde{e}^{-} \tilde{e}^{-}$is produced, with cross section being proportional to a mere single power of the velocity, $\beta_{\tilde{e}}$ [29]. Produced $\tilde{e}^{-}$decays into the NLSP $\tilde{\tau}$ with velocity $\beta_{\tilde{\tau}}$, which does not differ much from $\beta_{\tilde{e}}$ as long as the mass difference is small. Therefore, in order to produce many low velocity $\tilde{\tau}_{\mathrm{s}}$, the indirect production at a $e^{-} e^{-}$collider may be advantageous to the direct production at $e^{+} e^{-}$collider. Hence, in this section we investigate the possibility to study the CNLSP decay at a $e^{-} e^{-}$collider. A comparison between the $e^{-} e^{-}$and $e^{+} e^{-}$ cases will be given at the end of this section.

The selectron $\tilde{e}^{-}$can decay into both of $\tilde{\tau}^{-}$and $\tilde{\tau}^{+}$, mainly via three body decays $\tilde{e}^{-} \rightarrow$ $\tilde{\tau}^{-} \tau^{+} e^{-}$and $\tilde{e}^{-} \rightarrow \tilde{\tau}^{+} \tau^{-} e^{-}$, if these modes are kinematically allowed. ${ }^{6}$ In the following, we restrict ourselves to a pure "right-handed" NLSP stau $\tilde{\tau}_{R}$ and right-handed selectron $\tilde{e}_{R}$, for simplicity. We also assume that the modes $\tilde{e}^{-} \rightarrow \tilde{\tau}^{ \pm} \tau^{\mp} e^{-}$are kinematically allowed, i.e., $m_{\tilde{e}}-m_{\tilde{\tau}} \gtrsim 1.78 \mathrm{GeV}$. Then, for both the production and decay of the selectron, essentially only Bino exchange contributes. For simplicity, we assume that the lightest neutralino is a pure Bino, with mass $M_{1}>m_{\tilde{e}}$. Under those assumptions, energy and angular distributions of indirectly produced staus are determined only by 4 parameters, $m_{\tilde{\tau}}, m_{\tilde{e}}, M_{1}$, and $\beta_{\tilde{e}}$ (or equivalently the electron beam energy). Generalizations to cases beyond those assumptions will be straightforward. ${ }^{7}$

6 Those three-body decays of selectrons were studied in detail in Ref. [30].

${ }^{7}$ If the modes $\tilde{e}^{-} \rightarrow \tilde{\tau}^{ \pm} \tau^{\mp} e^{-}$are kinematically forbidden, the main mode is either $\tilde{e}^{-} \rightarrow \tilde{\tau}^{-} \bar{\nu}_{\tau} \nu_{e}$ or $\tilde{e}^{-} \rightarrow e^{-} \psi_{3 / 2}$ depending on the parameters. If the former is the main mode, only $\tilde{\tau}^{-}$will be collected. If the latter is dominant, $\tilde{e}^{-}$plays the role of NLSP and one can study the gravitino by its decay instead of 

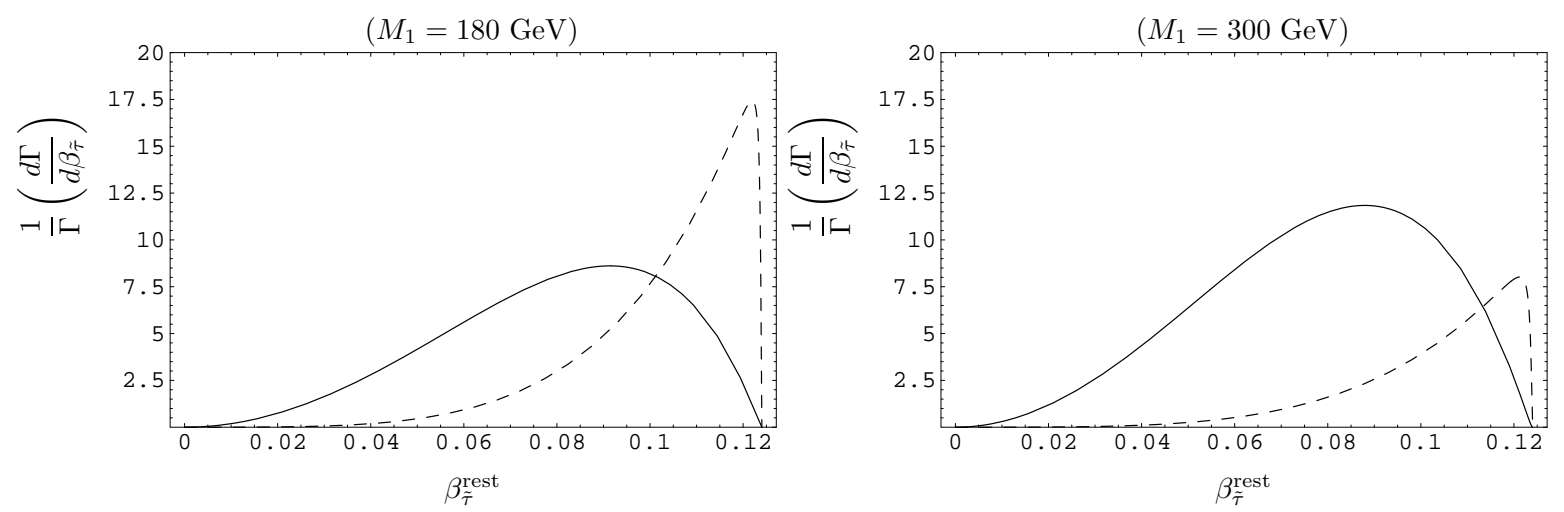

FIG. 3: Velocity distributions of $\tilde{\tau}^{+}$(solid line) and $\tilde{\tau}^{-}$(dashed line) at the rest frame of $\tilde{e}^{-}$, for $m_{\tilde{e}}=170 \mathrm{GeV}$ and $m_{\tilde{\tau}}=150 \mathrm{GeV}$. Bino mass is $M_{1}=180 \mathrm{GeV}$ (left) and $M_{1}=300 \mathrm{GeV}$ (right).

Production cross section of $\tilde{e}^{-} \tilde{e}^{-}$pair for $m_{\tilde{e}}=170 \mathrm{GeV}$ is shown in Fig. 2$]^{8}$ The angular distribution of $\tilde{e}^{-}$is almost isotropic for small $\beta_{\tilde{e}}$, and is mildly enhanced at beam direction for larger $\beta_{\tilde{e}}$. Notice that all the produced selectrons have the same velocity $\beta_{\tilde{e}}$ for a fixed beam energy. For instance, $\beta_{\tilde{e}}=0.4$ corresponds to a center-of-mass energy $E_{\mathrm{cm}}=371 \mathrm{GeV}$. Those selectrons decay into $\tilde{\tau}^{ \pm}$via three body decays $\tilde{e}^{-} \rightarrow \tilde{\tau}^{ \pm} \tau^{\mp} e^{-}$. Fig. [3] shows the velocity distributions of the produced $\tilde{\tau}^{ \pm}$at the rest frame of $\tilde{e}^{-}$, for $m_{\tilde{e}}=170 \mathrm{GeV}$ and $m_{\tilde{\tau}}=150 \mathrm{GeV}$. In this frame, $\tilde{\tau}^{ \pm}$are produced isotropically.

As can be seen in Fig. 3, there are interesting differences between $\tilde{\tau}^{+}$and $\tilde{\tau}^{-}$. First of all, the ratio of $\tilde{\tau}^{+} / \tilde{\tau}^{-}$events increases for larger $M_{1}[30]$. This is because the $\tilde{e}^{-} \rightarrow \tilde{\tau}^{+} \tau^{-} e^{-}$ mode picks up the Bino mass in the propagators (chirality flipped), while the other mode does not. Secondly, one can see that $\tilde{\tau}^{-}$distribution has a peak at larger velocity than $\tilde{\tau}^{+}$.

Now we can calculate the $(\eta, \beta)$ distributions of $\tilde{\tau}^{ \pm}$at the laboratory frame by combining the angular distribution of $\tilde{e}^{-}$with the energy distributions of $\tilde{\tau}^{ \pm}$as shown in Fig. 3. The results are shown in Fig. 4. for $m_{\tilde{\tau}}=150 \mathrm{GeV}, m_{\tilde{e}}=170 \mathrm{GeV}$, and $M_{1}=180 \mathrm{GeV}$. Here, we have normalized the total number of events so that it corresponds to integrated luminosity $10 \mathrm{fb}^{-1}$. One can see that the $\tilde{\tau}^{+}$distribution has a sharper peak at $\beta_{\tilde{\tau}} \simeq \beta_{\tilde{e}}$ than $\tilde{\tau}^{-}$. This is because $\tilde{\tau}^{+}$has typically smaller velocity than $\tilde{\tau}^{-}$at the rest frame of $\tilde{e}^{-}$, as can be seen in Fig. 3.

Finally, we estimate the total number of $\tilde{\tau}^{ \pm} \mathrm{S}$ which are stopped at a material. Here, as

$\tilde{\tau}$ 's decay.

${ }^{8}$ Here we have neglected the beam effect and finite width effects (cf. [31]). 

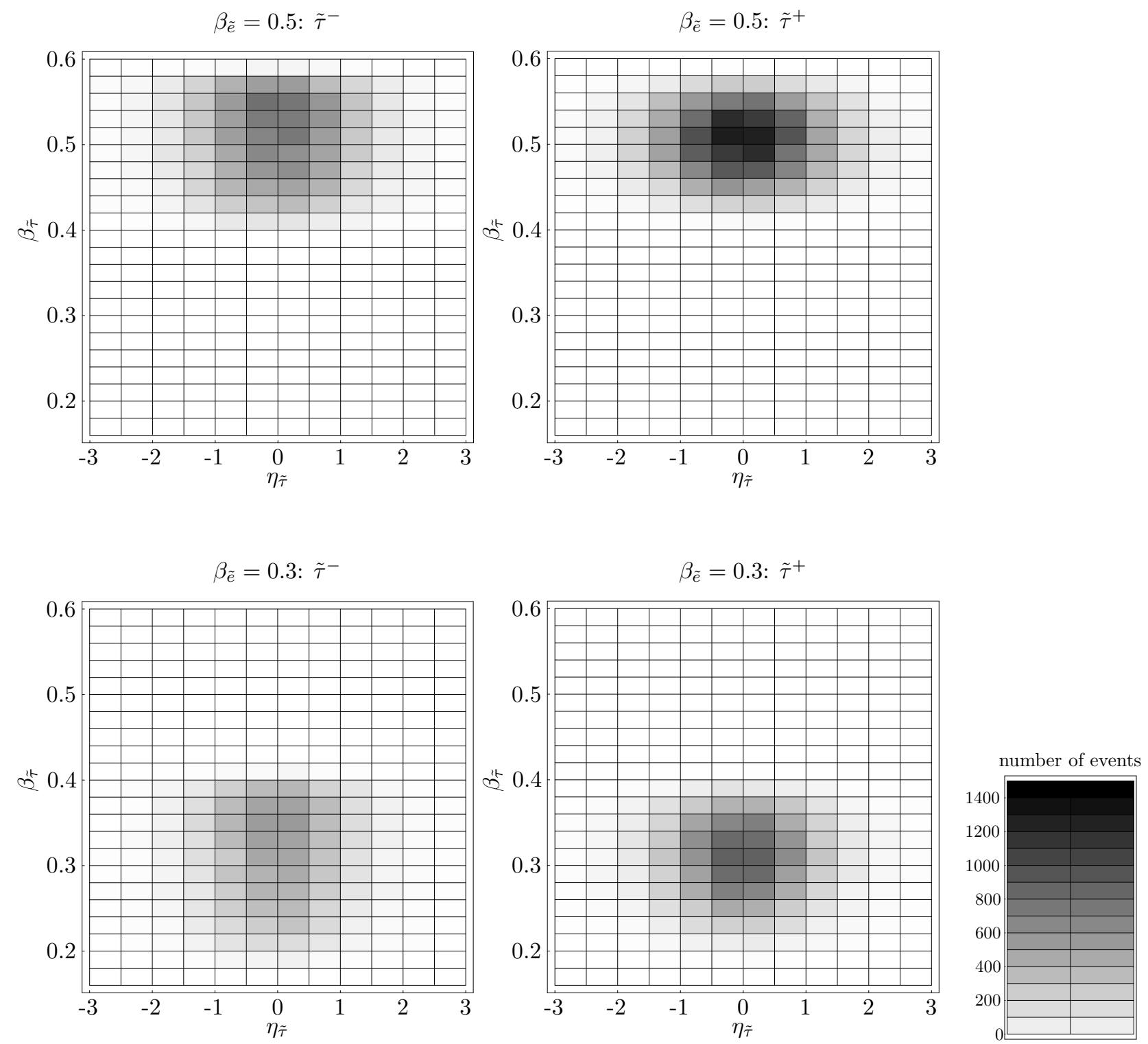

FIG. 4: $(\eta, \beta)$ distributions of $\tilde{\tau}^{ \pm}$at the laboratory frame, for $m_{\tilde{\tau}}=150 \mathrm{GeV}, m_{\tilde{e}}=170 \mathrm{GeV}$, and $M_{1}=180 \mathrm{GeV}$. The velocity of the selectron is $\beta_{\tilde{e}}=0.5$ for upper figures, and $\beta_{\tilde{e}}=0.3$ for lower figures. Total number of events are normalized so that it corresponds to integrated luminosity $10 \mathrm{fb}^{-1}$.

examples, we assume $1000 \mathrm{~g} / \mathrm{cm}^{2}, 3000 \mathrm{~g} / \mathrm{cm}^{2}$, and $5000 \mathrm{~g} / \mathrm{cm}^{2}$ of iron placed at the region $|\eta|<1$. For $m_{\tilde{\tau}}=150 \mathrm{GeV}$, they can stop staus with velocities $\beta_{\tilde{\tau}} \lesssim 0.37, \beta_{\tilde{\tau}} \lesssim 0.48$, and $\beta_{\tilde{\tau}} \lesssim 0.54$, respectively [12]. We have counted the number of events in these regions, for the parameter set used in Fig. 4. The results are shown in TableI

Given the weight of stopping material, one can tune the beam energy, thereby the selec- 


\begin{tabular}{|c|c|c|c|c|}
\hline & total & $1000 \mathrm{~g} / \mathrm{cm}^{2}$ & $3000 \mathrm{~g} / \mathrm{cm}^{2}$ & $5000 \mathrm{~g} / \mathrm{cm}^{2}$ \\
\hline & $\times 10^{4} \quad \times 10^{4}$ & $\times 10^{4} \quad \times 10^{4}$ & $\times 10^{4} \quad \times 10^{4}$ & $\times 10^{4} \quad \times 10^{4}$ \\
\hline$\beta_{\tilde{e}}=0.2$ & $1.47 \tilde{\tau}^{-} 1.83 \tilde{\tau}^{+}$ & $1.11 \tilde{\tau}^{-} 1.38 \tilde{\tau}^{+}$ & $1.11 \tilde{\tau}^{-} 1.38 \tilde{\tau}^{+}$ & $1.11 \tilde{\tau}^{-} 1.38 \tilde{\tau}^{+}$ \\
\hline$\beta_{\tilde{e}}=0.3$ & $2.06 \tilde{\tau}^{-} 2.56 \tilde{\tau}^{+}$ & $1.30 \tilde{\tau}^{-} 1.78 \tilde{\tau}^{+}$ & $1.54 \tilde{\tau}^{-} 1.90 \tilde{\tau}^{+}$ & $1.54 \tilde{\tau}^{-} 1.90 \tilde{\tau}^{+}$ \\
\hline$\beta_{\tilde{e}}=0.4$ & $2.47 \tilde{\tau}^{-} 3.08 \tilde{\tau}^{+}$ & $0.49 \tilde{\tau}^{-} 0.48 \tilde{\tau}^{+}$ & $1.71 \tilde{\tau}^{-} 2.22 \tilde{\tau}^{+}$ & $1.81 \tilde{\tau}^{-} 2.25 \tilde{\tau}^{+}$ \\
\hline$\beta_{\tilde{e}}=0.5$ & $2.67 \tilde{\tau}^{-} 3.33 \tilde{\tau}^{+}$ & $0 \tilde{\tau}^{-}$ & $0.60 \tilde{\tau}^{-} 0.65 \tilde{\tau}^{+}$ & $1.38 \tilde{\tau}^{-} 1.95 \tilde{\tau}^{+}$ \\
\hline
\end{tabular}

TABLE II: Estimated numbers of $\tilde{\tau}^{ \pm}$stopped in the material at $e^{-} e^{-}$collider with integrated luminosity $10 \mathrm{fb}^{-1}$. As a stopping material, we have assumed iron placed at $|\eta|<1$ region. Parameters are $m_{\tilde{\tau}}=150 \mathrm{GeV}, m_{\tilde{e}}=170 \mathrm{GeV}$, and $M_{1}=180 \mathrm{GeV}$.

tron velocity $\beta_{\tilde{e}}$, to maximize the obtained number of staus. We again assume that the total thickness which particle has to go through within the LC detector is about $1000 \mathrm{~g} / \mathrm{cm}^{2}$. The optimal velocity is $\beta_{\tilde{e}} \simeq 0.4$ for the stopper of the thickness $2000 \mathrm{~g} / \mathrm{cm}^{2}$, when $3.0 \times 10^{4}$ CNLSPs can be stopped in the stopper of $25 \mathrm{kton} \times\left(R_{\mathrm{IP}} / 10 \mathrm{~m}\right)^{2}$ for $10 \mathrm{fb}^{-1}$. For integrated luminosity $100 \mathrm{fb}^{-1}$ (corresponding to 250 days for $e^{-} e^{-}$luminosity in [32]) and a 10kton stopper, the total number of the CNLSP is therefore

$$
N=1.2 \times 10^{5}\left(\frac{M_{T} / 10 \mathrm{kton}}{\left(R_{\mathrm{IP}} / 10 \mathrm{~m}\right)^{2}}\right),
$$

for the present parameter set.

To illustrate the model dependence, let us consider another example, $\left(m_{\tilde{\tau}}, m_{\tilde{e}}, M_{1}\right)=$ $(100,103,110) \mathrm{GeV}$, where the masses are close to these for G2b point. This case is better than the previous one in several aspects. First of all, smaller $m_{\tilde{e}}$ and $M_{1}$ lead to a larger production cross section of $\tilde{e}$. Secondly, smaller $m_{\tilde{\tau}}$ means shorter range $R$ for a fixed velocity $\beta$. Finally, because of the small mass difference between $m_{\tilde{e}}$ and $m_{\tilde{\tau}}$, $\tilde{\tau}$ 's velocity has a very narrow distribution, $\left|\beta_{\tilde{\tau}}-\beta_{\tilde{e}}\right| \lesssim \beta_{\tilde{\tau}}^{\text {rest }} \simeq 0.024$. Assuming again $1000 \mathrm{~g} / \mathrm{cm}^{2}$ thickness of the LC detector at $|\eta|<1$, a $1000 \mathrm{~g} / \mathrm{cm}^{2}$ stopper will be sufficient to stop CNLSPs with velocity $0.4 \lesssim \beta_{\tilde{\tau}} \lesssim 0.48$. Therefore, for $\beta_{\tilde{e}}=0.44$, all the generated $\tilde{\tau}_{\mathrm{S}}\left(0.42<\beta_{\tilde{\tau}}<0.46\right)$ at $|\eta|<1$ will be stopped with a $12.6 \times\left(R_{\mathrm{IP}} / 10 \mathrm{~m}\right)^{2}$ kton stopper. We found that the total number of collected $\tilde{\tau}$ is

$$
N=0.9 \times 10^{6}\left(\frac{M_{T} / 10 \mathrm{kton}}{\left(R_{\mathrm{IP}} / 10 \mathrm{~m}\right)^{2}}\right)
$$


for integrated luminosity $100 \mathrm{fb}^{-1}$. Note that we have assumed a $1000 \mathrm{~g} / \mathrm{cm}^{2}$ stopper, and hence a wider area is covered for a fixed weight $M_{T}$ than the previous cases. For a 1kton stopper, roughly ten times more CNLSPs can be collected than Eq.(4).

So far we have assumed the thickness of the LC detector is uniform for $|\eta| \lesssim 1$. Ideally, given the masses $m_{\tilde{e}}$ and $m_{\tilde{\tau}}$, the total weight of the stopper $M_{T}$ and the profile of the collider detector, one can optimize the shape (thickness) of the stopper and the electron beam energy in order to maximize the collected number of CNLSPs.

With the above number of stopped CNLSP, one can measure the SUSY breaking scale from the lifetime very precisely. In addition, the mass of gravitino would be measured directly from the measurement of the upper end of the $\tau$ jet energy from the CNLSP decays. The end point is expressed as $E_{\tau} \sim\left(m_{\tilde{\tau}}^{2}-m_{3 / 2}^{2}\right) /\left(2 m_{\tilde{\tau}}\right)$. If the error of energy scale of the $\tau$ jet $\Delta E / E$ is $\epsilon$, a gravitino mass of $m_{3 / 2} \gtrsim \sqrt{\epsilon} \times m_{\mathrm{NLSP}}$ can be measured. For example, for $m_{\tilde{\tau}}=150 \mathrm{GeV}$ and $m_{3 / 2}=30 \mathrm{GeV}^{9}$, the tau energy is $72 \mathrm{GeV}$. If it is measured within $\epsilon=3 \%$, gravitino mass will be determined as $m_{3 / 2}=(16-39) \mathrm{GeV}$. The lifetime $\tau_{\mathrm{CNLSP}}=95$ days and the mass $m_{\tilde{\tau}}$ will be measured much more accurately. Notably this leads to a measurement of Planck scale $M_{\mathrm{P}}=(1.9-4.6) \times 10^{18} \mathrm{GeV}$ by using Eq. (11).

We found that there might be $\mathrm{O}\left(10^{5}\right)$ events available for the CNLSP study if $e^{-} e^{-}$linear collider can produce $\tilde{e}^{-} \tilde{e}^{-}$near the threshold and $\tilde{e}^{-}$decays into a CNLSP which is close to $\tilde{e}^{-}$in mass. When the lifetime of the gravitino is order of a year or less, all of the stopped CNLSP decay will be observed, and about $1 \%$ of them decay through its rare decay mode such as $\tilde{\tau} \rightarrow \gamma \tau \psi_{3 / 2}$.

In paper [11], the importance to study such rare decay modes is discussed. The decay into gravitino is induced by the interaction

$$
L_{3 / 2}=-\frac{1}{\sqrt{2} M_{\mathrm{P}}}\left[\left(D_{\nu} \tilde{\tau}_{R}\right)^{*} \bar{\psi}^{\mu} \gamma^{\nu} \gamma_{\mu} P_{R} \tau+\left(D_{\nu} \tilde{\tau}_{R}\right) \bar{\tau} P_{L} \gamma_{\mu} \gamma^{\nu} \psi^{\mu}\right]+(L \leftrightarrow R)
$$

In the limit of very large gaugino masses, the 3 body decay proceeds through the photon emission from the any of the charged external lines of the decay $\tilde{\tau} \rightarrow \psi_{3 / 2} \tau$, or the 4 point photon-gravitino-tau-stau interactions.

The peculiarity of the stau decay into the gravitino compared to those by ordinary Yukawa type interaction may be seen more clearly in the limit $m_{\tilde{\tau}} \gg m_{3 / 2}$ when the helicity $1 / 2$

\footnotetext{
${ }^{9}$ These masses are (marginally) consistent with the BBN constraint on the NLSP decay [4].
} 
component dominates the gravitino interaction, because the wave function of $h=1 / 2$ components will be enhanced proportional to its energy. Indeed it is reasonable to assume this limit for the rare mode study, because the CNLSP lifetime should not be too much larger than $\mathrm{O}$ (year) to make a full use of the all stopped CNLSPs. This means the gravitino mass cannot be comparable to the stau mass, in that case lifetime easily exceeds $\mathrm{O}$ (year).

In the limit $m_{\tilde{\tau}} \gg m_{3 / 2}$, the $\tilde{\tau}$ decay then may be governed by the effective interaction of goldstino $\chi$ where $\psi_{\mu} \sim \sqrt{2 / 3}\left(\partial_{\mu} \chi\right) / m_{3 / 2}$,

$$
L_{\mathrm{eff}}=\frac{m_{\tilde{\tau}}^{2}}{\sqrt{3} M_{\mathrm{P}} m_{3 / 2}}\left(\tilde{\tau}_{R}^{*} \bar{\chi} P_{R} \tau+\tilde{\tau}_{R} \bar{\tau} P_{L} \chi\right)+(L \leftrightarrow R)-\frac{m_{\tilde{\gamma}}}{4 \sqrt{6} M_{\mathrm{P}} m_{3 / 2}} \bar{\chi}\left[\gamma^{\mu}, \gamma^{\nu}\right] \tilde{\gamma} F_{\mu \nu}
$$

The first term is Yukawa type interaction and renormalizable, while the last term (photonphotino-gravitino interaction) is a non-renormalizable one. The photon-photino-gravitino interaction contributes to the CNLSP decay through the photino exchange. The last term manifests itself in the three body decay distribution of gravitinos, and may be extracted from the study of the decay distribution.

The difference between the decay pattern of the gravitino to some fermion, which comes from a non-renormalizable terms, may be studied by measuring the photon energy and angle between photon and $\tau$ [11]. For the type of the drift tube detector discussed in the previous section, the angle between the tau jets and photon would be measured precisely. The energy resolution, on the other hand, would be around $10 \%$ event by event. The statistical significance of the measurement of the effective couplings in Eq. (92) at a future linear collider will be studied elsewhere.

Before closing this section, let us compare the cases of $e^{+} e^{-}$and $e^{-} e^{-}$colliders. As noted before, the production cross sections $\sigma\left(e^{+} e^{-} \rightarrow \tilde{l}_{R}^{+} \tilde{l}_{R}^{-}\right)$are suppressed by $\beta^{2}$ compared to $\sigma\left(e^{-} e^{-} \rightarrow \tilde{e}_{R}^{-} \tilde{e}_{R}^{-}\right)$, where $\tilde{l}_{R}=\tilde{\tau}_{R}, \tilde{\mu}_{R}, \tilde{e}_{R}$. Quantitatively, they are even more suppressed. We have numerically checked that $\sigma\left(e^{+} e_{R}^{-} \rightarrow \tilde{\tau}_{R}^{+} \tilde{\tau}_{R}^{-}\right) / \sigma\left(e_{R}^{-} e_{R}^{-} \rightarrow \tilde{e}_{R}^{-} \tilde{e}_{R}^{-}\right)<0.015$ and $\sigma\left(e^{+} e_{R}^{-} \rightarrow\right.$ $\left.\tilde{e}_{R}^{+} \tilde{e}_{R}^{-}\right) / \sigma\left(e_{R}^{-} e_{R}^{-} \rightarrow \tilde{e}_{R}^{-} \tilde{e}_{R}^{-}\right)<0.014$ for $\beta<0.5$, for both of the above examples $\left(m_{\tilde{\tau}}, m_{\tilde{e}}, M_{1}\right)=$ $(150,170,180) \mathrm{GeV}$ and $(100,103,110) \mathrm{GeV}$. Even for unpolarized electron beam, the ratios are less than 0.037. On the other hand, the luminosity of a $e^{-} e^{-}$collider becomes smaller than $e^{+} e^{-}$due to the de-focusing effect of the same charge beams. It is found in Ref. 32] that, assuming identical beam parameters, the luminosity of $e^{-} e^{-}$mode is seven times smaller than the $e^{+} e^{-}$mode. Therefore, $e^{-} e^{-}$mode can produce at least four times more low velocity CNLSP staus than $e^{+} e^{-}$, per time. 
Another possibility is a $\tilde{e}_{R}^{ \pm} \tilde{e}_{L}^{\mp}$ pair production at $e^{+} e^{-}$collider, at the threshold $E_{\mathrm{cm}} \simeq$ $m_{\tilde{e}_{R}}+m_{\tilde{e}_{L} \cdot}{ }^{10}$ Although $\sigma\left(e^{+} e^{-} \rightarrow \tilde{e}_{R}^{+} \tilde{e}_{R}^{-}\right)$is suppressed by $\beta^{3}, \sigma\left(e^{+} e^{-} \rightarrow \tilde{e}_{R}^{ \pm} \tilde{e}_{L}^{\mp}\right)$ is suppressed only by $\beta$. In the limit of $m_{\tilde{e}_{L}} \simeq m_{\tilde{e}_{R}}$ and $\beta \ll 1, \sigma\left(e_{R}^{+} e_{R}^{-} \rightarrow \tilde{e}_{L}^{+} \tilde{e}_{R}^{-}\right)=\sigma\left(e_{L}^{+} e_{L}^{-} \rightarrow \tilde{e}_{R}^{+} \tilde{e}_{L}^{-}\right)$is eight times smaller than $\sigma\left(e_{R}^{-} e_{R}^{-} \rightarrow \tilde{e}_{R}^{-} \tilde{e}_{R}^{-}\right)$, because of the bino coupling $(\times 1 / 4)$, absence of $u$-channel $(\times 1 / 4)$ and non-identical final states $(\times 2)$. This factor offsets the advantage of the luminosity. Besides, $\tilde{e}_{L}$ is typically heavier than $\tilde{e}_{R}$ by $\mathrm{O}(1)$ factor. This leads to additional suppression of the cross section $\sigma \propto M_{1}^{2} / E_{\mathrm{cm}}^{4}$ by a factor of $\left(2 m_{\tilde{e}_{R}} /\left(m_{\tilde{e}_{L}}+m_{\tilde{e}_{R}}\right)\right)^{4}$. A CNLSP generated by $\tilde{e}_{L}$ is too rapid to stop in the stopper, resulting in another suppression of factor $1 / 2$. Therefore, the $e^{-} e^{-}$option is still likely advantageous to the $e^{+} e^{-}$option.

\section{DISCUSSION}

In this paper we have discussed the possibility to study the charged NLSP (CNLSP) $\tilde{l}$ decaying into the LSP gravitino at future collider experiments such as the LHC or $e^{+} e^{-} / e^{-} e^{-}$ linear colliders. $\mathrm{O}\left(10^{4}\right)(\mathrm{LHC})$ to $\mathrm{O}\left(10^{5}\right)$ (LC) CNLSP may be stopped if a 1 kton stopper is placed close to the detectors at the interaction point for the case where sparticle masses are close to the current experimental limits.

A CNLSP lifetime up to $10^{3}$ years will be accessible when $10^{4}$ CNLSPs are stopped in the stopper, because more than 10 CNLSPs decay inside the detector/year in that case. If the CNLSP production rate is low and their lifetime is too long, the signal to background ratio could be problematical. In such a serious case, we might be forced to do detection only during beam-off period, with cost of lower detection efficiency. Detail estimation of the signal to background ratio needs further simulations with realistic detector design and experimental situations.

For $\mathrm{O}\left(10^{5}\right)$ CNLSPs, one can study the rare decays of the CNLSP. It should be noted that not only the gravitino-CNLSP interaction, but also all the other exotic interactions involving CNLSP can be investigated by collecting metastable CNLSP.

In this paper we restrict ourselves to the case where the long-lived charged particle is the CNLSP $\tilde{\tau}$. Stable gluino scenario [33, 34] or split SUSY scenario where the gluino lifetime is long because of superheavy sfermions [35] predict a hadronic charged stable or

${ }^{10}$ We thank P. M. Zerwas for pointing out this option. 
meta-stable particle. They may also be stopped by a massive stopper detector. In the case of split SUSY scenario, the gluino lifetime depends on the superheavy sfermion mass scale. However, the number of stopped gluinos would be significantly smaller than those expected for the case where $m_{\mathrm{CNLSP}} \sim 100 \mathrm{GeV}$ studied in this paper, because the stopping range increases proportional to the masses.

The physics that can be extracted from the lifetime measurement is rich. One can determine the scale of the hidden sector SUSY breaking by measuring the NLSP lifetime $\tau_{\mathrm{NLSP}}$. This restricts the possible SUSY breaking scenario strongly. The direct information on the NLSP lifetime also constrains the possible cosmological scenario. If the NLSP lifetime is longer than $\mathrm{O}(1)$ sec, the decay of NLSP may change the abundances of the light elements of nuclei in the early Universe, so that the upper limit on the number density at the time of NLSP decay in the early Universe would be obtained [3]. This in turn severely restricts the parameters of supersymmetric models [4, 5].

If the CNLSP turns out to be long-lived, high priority must be given to the study of the CNLSP lifetime so that the measurement can be done during the high luminosity run of the LHC. Suitable 1kton detectors exist already for proton decay searches/neutrino detector now, and we discussed the Soudan 2 detector as an example. It is important to keep these detectors after their original physics target is achieved by the time when the physics results at the low luminosity run is obtained at the LHC.

\section{Note added}

After this paper appeared in e-print archive, Feng and Smith also submitted a paper 36] on the number of slepton that would be trapped at the stopper near the LHC and LC. They consider the possibility to place a water tank outside the main detector, then drain the water to a reservoir in low background environment. This perhaps is not useful for the NLSP with lifetime much shorter than draining period.

\section{acknowledgment}

We thank A. Ibarra, M. Ratz, F. D. Steffen, S. Tanaka and P. M. Zerwas for valuable discussions. K.H. thanks J. L. Feng and M. Sher for the exciting conversation at the SUSY'04 
conference. The authors thank the Yukawa Institute for Theoretical Physics at Kyoto University, where this work was initiated during the YITP-W-04-04 workshop on "Progress in Particle Physics". This work is supported in part by the Grant-in-Aid for Science Research, Ministry of Education, Science and Culture, Japan (No.14540260 and 14046210 for M.M.N.). M.M.N. is also supported in part by a Grant-in-Aid for the 21st Century COE "Center for Diversity and Universality in Physics".

[1] T. Moroi, H. Murayama and M. Yamaguchi, Phys. Lett. B 303 (1993) 289.

[2] M. Bolz, W. Buchmuller and M. Plumacher, Phys. Lett. B 443 (1998) 209 arXiv:hep-ph/9809381,

M. Fujii and T. Yanagida, Phys. Lett. B 549 (2002) 273 arXiv:hep-ph/0208191,

W. Buchmuller, K. Hamaguchi and M. Ratz, Phys. Lett. B 574 (2003) 156 arXiv:hep-ph/0307181,

M. Fujii, M. Ibe and T. Yanagida, Phys. Rev. D 69 (2004) 015006 arXiv:hep-ph/0309064,

M. Ibe and T. Yanagida, Phys. Lett. B 597 (2004) 47 arXiv:hep-ph/0404134.

[3] See, for recent detailed analysis, M. Kawasaki, K. Kohri and T. Moroi, arXiv:astro-ph/0402490, arXiv:astro-ph/0408426.

[4] J. L. Feng, S. Su and F. Takayama, arXiv:hep-ph/0404231.

[5] J. R. Ellis, K. A. Olive, Y. Santoso and V. C. Spanos, Phys. Lett. B 588 (2004) 7 arXiv:hep-ph/0312262; arXiv:hep-ph/0408118,

F. Wang and J. M. Yang, arXiv:hep-ph/0405186

L. Roszkowski and R. R. de Austri, arXiv:hep-ph/0408227

[6] J. L. Feng, A. Rajaraman and F. Takayama, Phys. Rev. Lett. 91 (2003) 011302 arXiv:hep-ph/0302215; Phys. Rev. D 68 (2003) 063504 arXiv:hep-ph/0306024.

[7] R. Allahverdi and M. Drees, arXiv:hep-ph/0408289.

[8] M. Drees and X. Tata, Phys. Lett. B 252, 695 (1990).

[9] J. L. Feng and T. Moroi, Phys. Rev. D 58, 035001 (1998) arXiv:hep-ph/9712499,

S. P. Martin and J. D. Wells, Phys. Rev. D 59, 035008 (1999) arXiv:hep-ph/9805289.

[10] S. Ambrosanio and G. A. Blair, Eur. Phys. J. C 12, 287 (2000) arXiv:hep-ph/9905403,

S. Ambrosanio, B. Mele, S. Petrarca, G. Polesello and A. Rimoldi, JHEP 0101, 014 (2001) 
arXiv:hep-ph/0010081,

P. G. Mercadante, J. K. Mizukoshi and H. Yamamoto, Phys. Rev. D 64 (2001) 015005 arXiv:hep-ph/0010067.

[11] W. Buchmuller, K. Hamaguchi, M. Ratz and T. Yanagida, Phys. Lett. B 588, 90 (2004) arXiv:hep-ph/0402179; arXiv:hep-ph/0403203.

[12] S. Eidelman et al. [Particle Data Group Collaboration], Phys. Lett. B 592, 1 (2004).

[13] J. L. Goity, W. J. Kossler and M. Sher, Phys. Rev. D 48 (1993) 5437 arXiv:hep-ph/9305244.

[14] J. L. Feng, A. Rajaraman and F. Takayama, arXiv:hep-th/0405248,

J. L. Feng, talk presented at the SUSY'04 conference, Tsukuba, Japan, June 17-23, 2004,

J. L. Feng, private communication, at the SUSY'04 conference.

[15] "ATLAS: Detector and physics performance technical design report. Volume 1," CERNLHCC-99-14

[16] "ATLAS detector and physics performance. Technical design report. Vol. 2,"CERN-LHCC$99-15$

[17] http://cmsinfo.cern.ch/Welcome.html, and documents there in.

[18] M. Dine, A. E. Nelson, Y. Nir and Y. Shirman, Phys. Rev. D 53, 2658 (1996) arXiv:hep-ph/9507378,

See also, G. F. Giudice and R. Rattazzi, Phys. Rept. 322, 419 (1999) arXiv:hep-ph/9801271.

[19] J. A. Aguilar-Saavedra et al. [ECFA/DESY LC Physics Working Group Collaboration], arXiv:hep-ph/0106315,

K. Abe et al. [ACFA Linear Collider Working Group Collaboration], arXiv:hep-ph/0109166.

T. Abe et al. [American Linear Collider Working Group Collaboration], in Proc. of the APS/DPF/DPB Summer Study on the Future of Particle Physics (Snowmass 2001) ed. N. Graf, SLAC-R-570 Resource book for Snowmass 2001, 30 Jun - 21 Jul 2001, Snowmass, Colorado

[20] M. M. Nojiri, K. Fujii and T. Tsukamoto, Phys. Rev. D 54, 6756 (1996) arXiv:hep-ph/9606370.

[21] F. E. Paige, S. D. Protopescu, H. Baer and X. Tata, arXiv:hep-ph/0312045.

[22] I. Hinchliffe and F. E. Paige, Phys. Rev. D 60, 095002 (1999) arXiv:hep-ph/9812233.

[23] G. Corcella et al., JHEP 0101, 010 (2001) arXiv:hep-ph/0011363.

[24] T. Toshito [Super-Kamiokande Collaboration], arXiv:hep-ex/0105023 
[25] M. Honda, T. Kajita, K. Kasahara and S. Midorikawa, arXiv:astro-ph/0404457

[26] ATLAS collaboration, Muon Spectrometer Technical Design Report, CERN/LHCC/9722(1997).

[27] B. C. Allanach et al., Eur. Phys. J. C 25, 113 (2002) [eConf C010630, P125 (2001)] arXiv:hep-ph/0202233.

[28] W. W. M. Allison et al. [Soudan-2 Collaboration], Nucl. Instrum. Meth. A 376, 36 (1996). Nucl. Instrum. Meth. A 381, 385 (1996).

[29] J. L. Feng, Int. J. Mod. Phys. A 13, 2319 (1998) arXiv:hep-ph/9803319.

[30] S. Ambrosanio, G. D. Kribs and S. P. Martin, Nucl. Phys. B 516 (1998) 55 arXiv:hep-ph/9710217.

[31] J. L. Feng and M. E. Peskin, Phys. Rev. D 64 (2001) 115002 arXiv:hep-ph/0105100,

A. Freitas, D. J. Miller and P. M. Zerwas, Eur. Phys. J. C 21 (2001) 361 arXiv:hep-ph/0106198,

A. Freitas, A. von Manteuffel and P. M. Zerwas, Eur. Phys. J. C 34 (2004) 487 arXiv:hep-ph/0310182.

[32] R. Brinkmann, K. Flottmann, J. Rossbach, P. Schmuser, N. Walker and H. Weise, TESLA Technical design report. Pt. 2: The accelerator, DESY-01-011.

[33] C. H. Chen, M. Drees and J. F. Gunion, Phys. Rev. D 55, 330 (1997) [Erratum-ibid. D 60, 039901 (1999)] arXiv:hep-ph/9607421.

[34] H. Baer, K. m. Cheung and J. F. Gunion, Phys. Rev. D 59, 075002 (1999) arXiv:hep-ph/9806361.

[35] N. Arkani-Hamed and S. Dimopoulos, arXiv:hep-th/0405159

[36] J. L. Feng and B. T. Smith, arXiv:hep-ph/0409278 